Supporting Information

\title{
Multilevel Optical Labeling by Spectral Luminescence Control in Nanodiamond Color Centers
}

Yan-Ting Chuang, Sheng-Ding Chen, Wei-Chun Huang, Tien-Lin Shen, Ming-Shien Chang, Yang-Fang Chen, Ya-Ping Hsieh*, Yuan-Huei Chang* and Mario Hofmann*

\section{Details of Cathodoluminescence spectra}

From the CL spectrum, we observed a series emission from N3 aggregate center, revealing high defect density of nitrogen atoms and vacancy numbers. Several peaks can be assigned in Figure 1a: NV centers contribute a strong zero-phonon line (ZPL) at $575 \mathrm{~nm}$ and a high intensity phonon side-band around $600 \mathrm{~nm}$. In addition to the N3 phonon side-band at $445 \mathrm{~nm}$ a faint $\mathrm{ZPL}$ is visible at $415 \mathrm{~nm} .{ }^{1}$ Peaks around $496 \mathrm{~nm}$ are thought to originate from $\mathrm{H} 3$ and $\mathrm{H} 4$ centers. $^{2}$ The emission located around $290 \mathrm{~nm}$ was identified as N4 center. $^{3}$

\section{Size dependence of fluorescence spectrum.}

The fluorescence of nanodiamond samples with different particle sizes $(35 \mathrm{~nm}, 100 \mathrm{~nm}, 350 \mathrm{~nm}, 700 \mathrm{~nm}$ diameter) were investigated. All samples exhibit emission from both N3 and NV centers. The relative intensity of the two emission peaks, however, was found to change significantly with diameter, revealing different concentrations of $\mathrm{N} 3$ center. 
The sized dependence is thought to be related to the density of surface defects. The surface of fNDs forms a graphitic layer of a few nanometer thickness which assist in the formation of defects. ${ }^{4}$ Due to their high surface-volume ratio, smaller fNDs will thus exhibit higher concentrations of N3 defects.

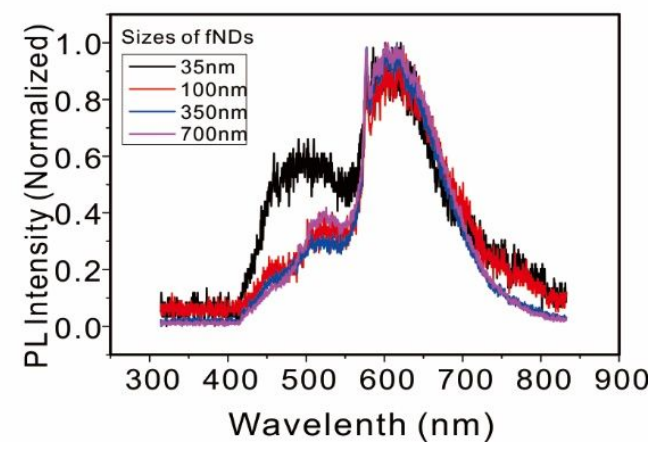

Figure S1. PL spectra of fNDs with different size

\section{Time-scales for internal photoconversion}

The N3 photoluminescence bleaching time in Figure 2a exhibits a time constant of approximately 5s. This is much slower than the time scales for nuclear or electronic spin transitions. ${ }^{5}$

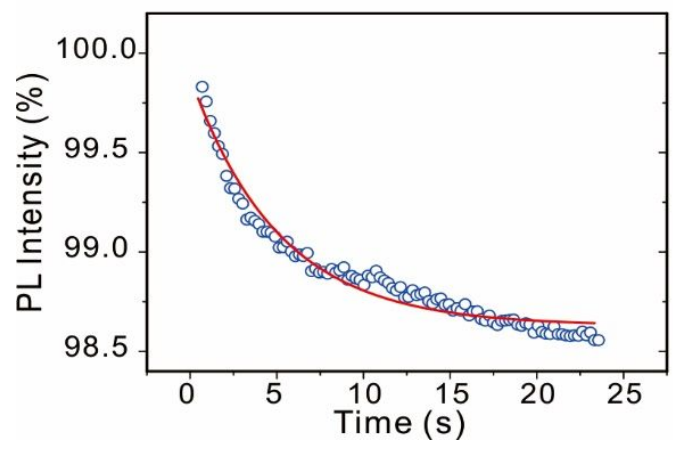

Figure S2. Exponential fit to transient PL intensity demonstrating lower fitting quality than diffusion model in the manuscript and revealing 5 s decay time 


\section{Derivation of diffusion coefficient from photoluminescence}

Assuming low-level injection conditions in a doped semiconductor, the recombination rate depends on the concentration of minority carriers available at the location of the laser illumination. We consider a simple diffusion picture and obtain a Green's function for minority carriers ${ }^{6}$ :

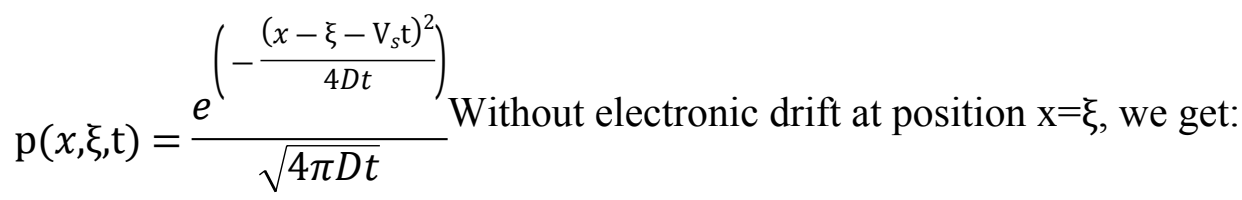

$$
\mathrm{p}(\mathrm{t})=\frac{p_{0}}{\sqrt{4 \pi D t}}
$$

\section{Derivation of diffusion coefficient from transient illumination}

For particle size larger than diffusion length ${ }^{7}$ :

$$
\tau_{e f f}=\tau_{b u l k} \times \frac{\sqrt{\frac{D}{\tau_{b u l k}}}}{\sqrt{\frac{D}{\tau_{b u l k}}}+S_{t o p}}
$$

where $\mathrm{D}$ is carrier diffusion coefficient, $\mathrm{S}_{\text {top }}$ is carrier recombination velocity, and $\tau_{\text {eff }}$ and $\tau_{\text {bulk }}$ are effective and bulk carrier lifetime.

From the definition of quantum efficiency $\eta$ :

$$
\eta=\frac{\mathrm{I}_{\mathrm{PL}}}{\mathrm{I}_{\text {LASER }}}=\frac{\tau_{r}}{\tau_{r}+\tau_{e f f}}
$$

Where $\tau_{r}$ is radiative lifetime, $\mathrm{I}_{\mathrm{PL}}$ and $\mathrm{I}_{\mathrm{LASER}}$ are PL intensity and excited laser power.

Then replacing $\tau$ in terms of $\eta$ : 


$$
\begin{gathered}
\mathrm{D}=\tau_{b} \times\left(\frac{S_{\text {top }} \times \tau_{r} \times \frac{I_{\text {LASER }}-I_{P L}}{I_{P L}}}{\tau_{b}-\tau_{r} \times \frac{I_{L A S E R}-I_{P L}}{I_{P L}}}\right)^{2} \\
\Rightarrow \mathrm{D}=\tau_{b} \times\left(\frac{S_{\text {top }} \times \tau_{r} \times\left(\frac{1}{\eta}-1\right)}{\tau_{b}-\tau_{r} \times\left(\frac{1}{\eta}-1\right)}\right)^{2}
\end{gathered}
$$

\section{Raw data for Figure 3c}

The PL spectra from which the peak intensities were extracted are provided in Figure S3.

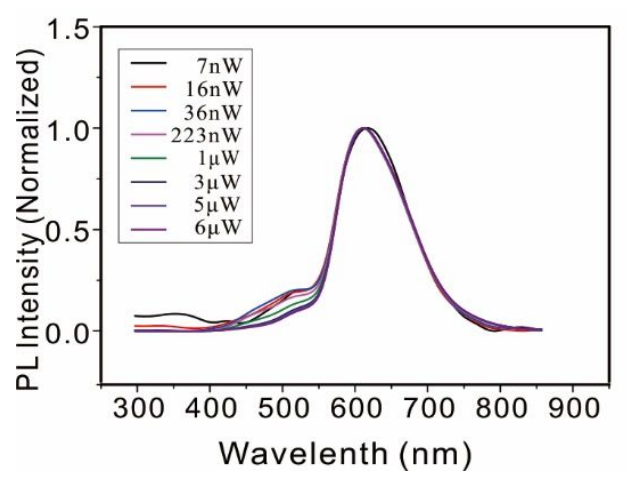

Figure S3. PL spectra of fND at different excitation power

\section{Investigation of alternative writing mechanisms}

To investigate alternative explanations, such as Joule-heating or electric field effects, we conduct two

alternative treatments of the samples. One is annealing under $500^{\circ} \mathrm{C}$ with $10 \mathrm{sccm}_{2}$ gas to reduce lattice defects and re-arrange lattice mismatch inside fNDs. And the other is treated with $10 \mathrm{~mW}-532 \mathrm{~nm}$ laser for 10s. The longer wavelength of excited laser must heat up the sample instead of exciting electrons to higher 
energy level. Their results of PL spectrum are showed in Figure S4(red for $\mathrm{H}_{2}$ annealing) and Figure

S5(green for irradiation treatment). We notice that both of two sample do not exhibit a changed spectrum compared to untreated fNDs(black).

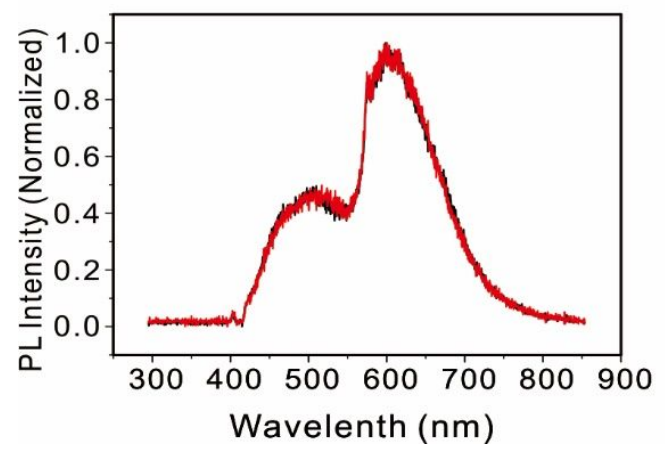

Figure S4. Comparison of PL spectra before and after annealing treatment

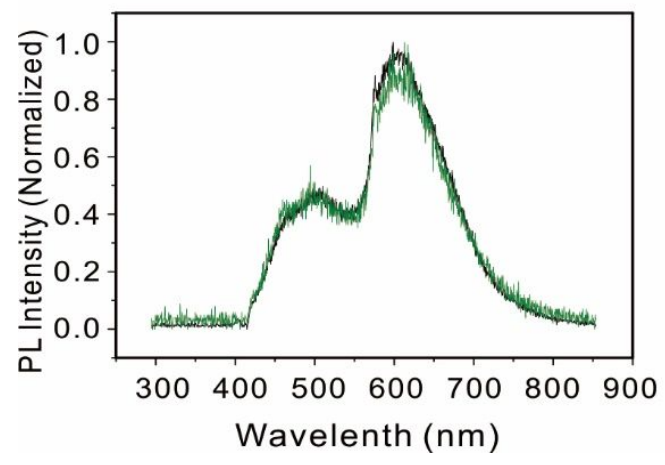

Figure S5. Comparison of PL spectra before and after high-intensity exposure to a 532nm laser

\section{REFERENCE}

(1) Hui, Y. Y.; Chang, H. C. Recent Developments and Applications of Nanodiamonds as Versatile

Bioimaging Agents. Journal of the Chinese Chemical Society 2014, 61 (1), 67-76. 
(2) De Sa, E.; Davies, G. Uniaxial Stress Studies of the 2.498 Ev (H4), 2.417 Ev and 2.536 Ev Vibronic

Bands in Diamond. Proceedings of the Royal Society of London. A. Mathematical and Physical Sciences

1977, 357 (1689), 231-251.

(3) Dischler, B. Handbook of Spectral Lines in Diamond: Volume 1: Tables and Interpretations, Springer

Science \& Business Media: 2012; Vol. 1.

(4) Mochalin, V. N.; Shenderova, O.; Ho, D.; Gogotsi, Y. The Properties and Applications of

Nanodiamonds. Nature nanotechnology 2012, 7 (1), 11.

(5) Collins, A.; Thomaz, M.; Jorge, M. I. B. Luminescence Decay Time of the 1.945 Ev Centre in Type Ib

Diamond. Journal of Physics C: Solid State Physics 1983, 16 (11), 2177.

(6) Blaj, G.; Kenney, C.; Segal, J.; Haller, G. Analytical Solutions of Transient Drift-Diffusion in Pn Junction Pixel Sensors. arXiv preprint arXiv:1706.01429 2017.

(7) Sameshima, T.; Nagao, T.; Yoshidomi, S.; Kogure, K.; Hasumi, M. Minority Carrier Lifetime Measurements by Photoinduced Carrier Microwave Absorption Method. Japanese Journal of Applied Physics 2011, 50 (3S), 03CA02. 